\title{
Correction to: Note orders suggest phrase-inserting structure in male Mueller's gibbon songs: a case study
}

\author{
Yoichi Inoue $^{1}$ - Waidi Sinun ${ }^{2} \cdot$ Shigeto Yosida ${ }^{3}$ Kazuo Okanoya ${ }^{1,4}$ \\ Published online: 8 June 2020 \\ (C) ISPA, CRL 2020
}

\section{Correction to: acta ethologica} https://doi.org/10.1007/s10211-020-00341-y

In the original version of this article, the Supplementary materials i.e., ESM 1, ESM 2 and ESM 6 should have been part of the article and presented in the Appendix section.

The original article has been corrected.

The online version of the original article can be found at https://doi.org/ 10.1007/s10211-020-00341-y

Yoichi Inoue

gibbonyoichi@hotmail.co.jp

Waidi Sinun

wsinun@ysnst.org.my

Shigeto Yosida

yoshida.shigeto@gmail.com

Kazuo Okanoya

cokanoya@mail.ecc.u-tokyo.ac.jp
Cognition and Behavior Joint Research Laboratory, RIKEN Brain Science Institute, 2-1 Hirosawa, Wako, Saitama 351-0198, Japan

2 Borneo Refugia Adventure SDN BHD, Level 12 Tun Mustapha Tower Likas Bay Sulaman Road, P. O. Box 11623, 88817 Kota Kinabalu, Sabah, Malaysia

3 IQVIA Solutions Japan K.K., Shinagawa Seaside South Tower, 4-12-1 Higashi-shinagawa, Shinagawa-ku, Tokyo 140-0002, Japan

4 Department of Life Sciences, Graduate School of Arts and Sciences, The University of Tokyo, 3-8-1 Komaba, Meguro-ku, Tokyo 153-8902, Japan 